\title{
Inhaled corticosteroids attenuate epithelial mesenchymal transition: implications for COPD and lung cancer prophylaxis
}

To the Editor:

We read with great interest the recent original research article by RAYMAKERS et al. [1] published in the European Respiratory Journal, regarding the beneficial effects of inhaled corticosteroids (ICS) in reducing the lung cancer risk in patients with chronic obstructive pulmonary disease (COPD). In their discussion, the authors mentioned that the mechanisms by which COPD is associated with an increased risk of lung cancer are not well-established. We do not know which mechanisms ICS inhibit for an appreciable reduction in lung cancer risk. This is a hugely important area and it is encouraging that leading respiratory journals are recognising this. Understanding these interactive mechanisms, we believe, is very important for better future translational approaches to establish early and preventive therapy [2, 3]. We would like to take this further and suggest a broader discussion on the latest findings in COPD pathology involving processes such as epithelial mesenchymal transition (EMT), angiogenesis and airway wall cellularity, and the effects of ICS on these aspects in COPD.

We have previously reported that EMT is an active process in both the small and large airways of smokers and patients with COPD, and has consequential effects on lung physiological parameters in these patients $[4,5]$. In the large airways, we observed that EMT-related changes were associated with increased hypervascularity of the underlying reticular basement membrane (Rbm), representing a typical active type-3 EMT process, considered as precursor to malignant conditions and metastasis [3]. It is important to note that it is in the large airways where most squamous cell carcinomas occur and type-3 EMT could be central to this [3]. The other key pathology associated with COPD is small airway or peri-bronchiolar fibrosis and obliteration, which is attributed to active type-2 EMT at this site, but fibrosis in general is also associated with malignancy $[3,5]$.

In a first proof-of-concept randomised controlled trial, we hypothesised that EMT might be the process through which this effect of ICS occurs. This study reported that inhaled fluticasone propionate delivered in high doses over 6 months did suppress airway epithelial activation and EMT-related changes in large airways of COPD patients [6]. In the same population, we also investigated effects of ICS on vascular remodelling in COPD [7]. However, ICS did not change Rbm vascularity but improved lamina propria vascularity in ex-smokers with COPD [7]. Physiological indices of air trapping showed negative correlations with increased vessel numbers, i.e. more vessels, less air trapping [7]. Perhaps for Rbm hypervascularity, longer duration with ICS or intravenous doses are required to attenuate vascular component of EMT.

More recently, in lung resections from cancer patients, we reported active EMT as the leading edge of invasive nonsmall cell lung cancer (both squamous cell and adenocarcinomas), with tumour aggressiveness strongly related to EMT activity [8]. Further, EMT markers within the tumours closely related to EMT activity from non-tumour-affected airway wall epithelium. This work suggests that the level of EMT activity in the airway wall, even in large airways that are acquiescent to bronchoscopic biopsy, could potentially be used as a marker for smokers most likely to develop both COPD and lung cancer. (EMT) in COPD http://bit.ly/2WG682I

Cite this article as: Lu W, Sharma P, Eapen MS, et al. Inhaled corticosteroids attenuate epithelial mesenchymal transition: implications for COPD and lung cancer prophylaxis. Eur Respir J 2019; 54: 1900778 [https://doi.org/10.1183/13993003.00778-2019]. 
Airway inflammation has also been suggested to be a contributor to lung cancer development in COPD, but careful interpretation is required. Our data from a comprehensive cross-sectional study demonstrated hypocellularity in the lamina propria of both large and small airway walls in COPD compared to never-smokers [9]. There was also no change in the proportions of key immune cell populations such as neutrophils, macrophages (CD68+), and CD8+ and CD4+ cells [9]. The only increase was observed for CD8+ cells in the small airways. We further identified differential macrophage switching in the small airway wall, lumen and alveolar spaces [10]. We observed that the airway wall in never-smokers was predominantly M2 (CD163+), which switched to a more M1 phenotype in COPD patients. In the lumen and alveolar spaces, however, we found an increase in M2 relative to M1 macrophages in both smokers and COPD patients, which was not seen in never-smokers. Bronchioalveolar lavage cytokine profile also matched these findings, and was skewed towards a more M2 profile [10]. Interestingly, M2 macrophages are also the predominant phenotype observed in lung cancer and they are known to promote tumorigenicity [3]. ICS may play a role in suppressing these specific phenotypic changes, thus restricting COPD progression to cancer, but this requires further investigation.

In summary, we believe that EMT with mucosal hypervascularity may represent fundamental important aspects of COPD pathology and a potential novel therapeutic target for prevention of both epithelial malignancy and fibrosis. ICS treatment does attenuate these changes, however, a safer drug than ICS would be ideal for long term use. Indeed, we may now be reaching a position that allows an integrated understanding of this airway disease, with the potential for this understanding to be translated into a new paradigm for earlier, or even preventive, therapy. It is vital to understand fundamental disease mechanisms for early interventions.

Wenying Lu, Pawan Sharma 1 , Mathew Suji Eapen and Sukhwinder Singh Sohal

Respiratory Translational Research Group, Dept of Laboratory Medicine, College of Health and Medicine, University of Tasmania, Launceston, Australia.

Correspondence: Sukhwinder Singh Sohal, Respiratory Translational Research Group, Dept of Laboratory Medicine, School of Health Sciences, College of Health and Medicine, University of Tasmania, Locked Bag - 1322, Newnham Drive, Launceston, Tasmania 7248, Australia. E-mail: sssohal@utas.edu.au

Received: April 182019 | Accepted: April 212019

Support statement: S.S. Sohal is supported by Clifford Craig Foundation Launceston General Hospital, Rebecca L. Cooper Medical Research Foundation, Lung Foundation Australia, Cancer Council Tasmania, Thoracic Society of Australia and New Zealand (TSANZ) and Boehringer Ingelheim. W. Lu is supported by Cancer Council Tasmania. P. Sharma is supported by Rebecca L. Cooper Medical Research Foundation, Australia.

Conflict of interest: None declared.

\section{References}

1 Raymakers AJN, Sadatsafavi M, Sin DD, et al. Inhaled corticosteroids and the risk of lung cancer in COPD: a population-based cohort study. Eur Respir J 2019; 53: 1801257.

2 Sohal SS, Walters EH. Advanced non-small-cell lung cancer. N Engl J Med 2017; 377: 1998-1999.

3 Eapen MS, Hansbro PM, Larsson-Callerfelt AK, et al. Chronic obstructive pulmonary disease and lung cancer: underlying pathophysiology and new therapeutic modalities. Drugs 2018; 78: 1717-1740.

4 Sohal S, Reid D, Soltani A, et al. Reticular basement membrane fragmentation and potential epithelial mesenchymal transition is exaggerated in the airways of smokers with chronic obstructive pulmonary disease. Respirology 2010; 15: 930-938.

5 Mahmood MQ, Sohal SS, Shukla SD, et al. Epithelial mesenchymal transition in smokers: large versus small airways and relation to airflow obstruction. Int J Chron Obstruct Pulmon Dis 2015; 10: 1515-1524.

6 Sohal SS, Soltani A, Reid D, et al. A randomized controlled trial of inhaled corticosteroids (ICS) on markers of epithelial-mesenchymal transition (EMT) in large airway samples in COPD: an exploratory proof of concept study. Int J Chron Obstruct Pulmon Dis 2014; 9: 533-542.

7 Soltani A, Walters EH, Reid DW, et al. Inhaled corticosteroid normalizes some but not all airway vascular remodeling in COPD. Int J Chron Obstruct Pulmon Dis 2016; 11: 2359-2367.

8 Mahmood MQ, Ward C, Muller HK, et al. Epithelial mesenchymal transition (EMT) and non-small cell lung cancer (NSCLC): a mutual association with airway disease. Med Oncol 2017; 34: 45.

9 Eapen MS, McAlinden K, Tan D, et al. Profiling cellular and inflammatory changes in the airway wall of mild to moderate COPD. Respirology 2017; 22: 1125-1132.

10 Eapen MS, Hansbro PM, McAlinden K, et al. Abnormal M1/M2 macrophage phenotype profiles in the small airway wall and lumen in smokers and chronic obstructive pulmonary disease (COPD). Sci Rep 2017; 7: 13392 . 